\title{
Easily detectable cytomorphological features to evaluate during ROSE for rapid lung cancer diagnosis: from cytology to histology
}

\author{
Sara Ravaioli ${ }^{1, *}$, Sara Bravaccini ${ }^{1, *}$, Maria Maddalena Tumedei ${ }^{1}$, Flavio Pironi ${ }^{2}$, \\ Piero Candoli ${ }^{3}$, Maurizio Puccetti² \\ ${ }^{1}$ Biosciences Laboratory, Istituto Scientifico Romagnolo per lo Studio e la Cura dei Tumori (IRST) IRCCS, Meldola, Italy \\ ${ }^{2}$ Pathology Unit, Santa Maria delle Croci Hospital, Ravenna, Italy \\ ${ }^{3}$ Pneumology Unit, Santa Maria delle Croci Hospital, Ravenna, Italy \\ *These authors have contributed equally to this work
}

Correspondence to: Sara Bravaccini, email: sara.bravaccini@irst.emr.it

Keywords: lung cancer, ROSE, histotype, cytomorphological features

Received: July 20, $2016 \quad$ Accepted: September 07, 2016 Published: November 08, 2016

\section{ABSTRACT}

In lung cancer patients, the only available diagnostic material often comes from biopsy or from cytological samples obtained by fine needle aspiration (FNA). There is a lack of easily detectable cytomorphological features for rapid on-site evaluation (ROSE) to orient lung cancer diagnosis towards a specific tumor histotype. We studied the cytological features evaluated on site to define tumor histotype and to establish the number of specimens to be taken. Cytological specimens from 273 consecutive patients were analyzed with ROSE: bronchoscopy with transbronchial needle aspiration (TBNA) had been performed in 72 patients and with endobronchial ultrasound (EBUS)-TBNA in 201. Cytomorphological features were correlated with the final diagnosis and diagnostic accuracy was measured. Analysis of the different cytomorphological parameters showed that the best sensitivity and specificity were obtained for adenocarcinoma by combining the presence of nucleoli and small/medium cell clusters, and for squamous cell carcinoma by considering the presence of necrosis $\geq \mathbf{5 0} \%$ and large cell clusters. For small cell carcinoma, the best diagnostic accuracy was obtained by combining moderate necrosis $(<50 \%)$ and the presence of single cells. Overall accuracy ranged from $90 \%$ to $97 \%$. We showed that it was possible to establish the histotype of the most frequent lung cancers during ROSE using only a few easily identifiable cytomorphological parameters. An accurate diagnosis during ROSE could help endoscopists to decide how many tumor samples must be taken, e.g.a higher number of samples is needed for the biomolecular characterization of adenocarcinoma, whereas one sample may be sufficient for squamous cell carcinoma.

\section{INTRODUCTION}

Lung cancer is the most common cause of death from cancer worldwide and only $30-40 \%$ of all lung cancers are operable $[1,2]$. The only available diagnostic material often comes from biopsy or from cytological samples obtained by fine needle aspiration (FNA). In the majority of cases, the final diagnosis is based on cytological evaluation and it is thus important to use the same biological material for molecular analysis [2-4].

Fine needle aspiration can be performed under radiological (conventional transbronchial needle aspiration
- TBNA) or ultrasound guidance (endobronchial ultrasound (EBUS)-TBNA) [5, 6]. The TBNA procedure is performed using a flexible fiberoptic bronchoscope to obtain tissue or fluid samples from the lungs and surrounding lymph nodes without conventional surgery [7]. EBUS is an invasive but highly effective procedure used to diagnose lung cancer, infections and other diseases causing enlarged lymph nodes in the chest [8].

The percentage of tumors diagnosed by cytological evaluation has increased since the advent of EBUSTBNA [8]. EBUS permits physicians to perform FNA on paratracheal and/or mediastinal lesions $[9,10]$. In fact, 
metastases in homo- and/or controlateral peribronchial and mediastinal lymph nodes can be more easily diagnosed and staged using this technique [1,9-11]. For inoperable tumors, cytology is the only valid tool for both diagnosis and biomolecular characterization [2-4].

Rapid on-site evaluation (ROSE) is a useful technique to rapidly assess the adequacy of biological material obtained by FNA [12-14] in terms of its cellularity. In eliminating unnecessary sampling, it also helps to reduce costs $[15,16]$. Given that ROSE is a widely used technique, different evaluation criteria have been proposed to determine specimen suitability for samples obtained by EBUS-TBNA, e.g. amount of lymphoid elements, presence of polluting bronchial cells, number of pigmented macrophages, amount of necrosis and presence of mucus and blood [15-18]. Jeffus et al. performed a comparison between Minnesota and New York criteria to establish the adequacy of EBUS-TBNA specimens [15], while Idowu and Powers analyzed the cytomorphological characteristics of primary lung cancers. In particular, typical cytomorphological features of adenocarcinoma include cellular clusters or acinar (glandular) arrangements, large vacuolated cells, high nuclear/cytoplasm (N/C) ratio, prominent nucleoli and finely granular chromatin [19]. From a cytomorphological point of view, well differentiated squamous cell carcinoma typically appears as individual cells or cohesive sheets of tumor cells, depending on the method of specimen procurement, with welldefined cell borders and dense cytoplasm. Small cell carcinomas typically consist of a biphasic population of viable and degenerating cells arranged in single or loose clusters with nuclear molding, individual cell necrosis and tumor diathesis. The viable tumor cells have a high $\mathrm{N} / \mathrm{C}$ ratio, smooth nuclear membranes, finely granular evenly dispersed chromatin and inconspicuous or absent nucleoli [19]. The morphological parameters used to determine sample adequacy for ROSE are the same as those of conventional cytology. However, they are timeconsuming, require the skills of an expert, and some are only visible with specific stains (e.g. chromatin with Papanicolau). There are still no easily detectable and reproducible cytomorphological features that can be used during ROSE to identify tumor histotype and facilitate the number of tissue samples to be taken, especially in adenocarcinoma where more samples are needed for biomolecular characterization. We thus decided to focus our attention on this issue.

The cytomorphological features recorded on site were retrospectively compared to the final diagnosis made on the formalin-fixed and paraffin-embedded (FFPE) material of the cytological specimen and further confirmed on the tumor tissue obtained by biopsy during broncoscopy and/or on resected tumor tissue from operable lesions. We also planned to evaluate the sensitivity and specificity of the identified criteria.

\section{RESULTS}

Histological diagnosis showed 131 (48\%) lung tumors and 22 (8\%) non-lung tumors. One hundred and twenty (44\%) samples were derived from non-tumor material (Table 1). Non-lung tumors were diagnosed exclusively by EBUS-TBNA. Samples obtained by TBNA were derived from lung tumors or normal lung tissue. Ninety $(45 \%)$ of the samples obtained by EBUS-TBNA were composed of reactive lymph node material (Table 1). $90 \%$ of the tumor samples obtained by EBUS-TBNA were from non-operable tumors that were metastatic at diagnosis.

In contrast, the majority of the peripheral lesions diagnosed by conventional TBNA were operable. Seventynine $(88 \%)$ of the 90 patients with reactive lymph nodes were followed up for 2 years. The initial diagnosis of reactive lymph nodes was confirmed in 74 (94\%) cases, while $5(6 \%)$ patients had a different final diagnosis: one adenocarcinoma, 3 sarcoidosis and one tuberculosis infection. Of the 14 specimens defined as non-tumoral by the cytological evaluation of TBNA material, 10 $(71 \%)$ were subsequently diagnosed as peripheral adenocarcinomas by CT-guided biopsy.

The distribution of cytomorphological features in relation to tumor histotype is shown in Figure 1. In particular, the presence of nucleoli and small/medium cell clusters was observed in $65(81 \%)$ and $72(90 \%)$ adenocarcinoma histotypes, respectively. The latter characteristic was almost always indicative of the presence of adenocarcinoma (Figure 2A, Figure 3A).

Squamous cell carcinomas were characterized by the presence of large cluster-forming cells and necrosis $\geq 50 \%$. The presence of large clusters was an exclusive characteristic of this tumor histotype (Figure 2B, Figure 3B). Finally, virtually all (95\%) of the small cell carcinomas showed $\geq 50 \%$ single cells (Figure 2C, Figure $3 \mathrm{C})$, while $84 \%$ showed moderate necrosis $(<50 \%)$.

Analysis of the different cytomorphological parameters revealed that the best sensitivity and specificity for adenocarcinoma were obtained by combining the presence of nucleoli and small/medium cell clusters. For squamous cell carcinoma, the presence of necrosis $\geq 50 \%$ and large clusters were considered as the best criteria for an accurate histological diagnosis, with an overall accuracy of $97 \%(P<0.001)$ determined by the chi-square test. Moreover, the best overall diagnostic accuracy of 94\% $(P<0.001)$ for small cell carcinoma was obtained by combining the characteristics of moderate necrosis $(<50 \%)$ and single cells (Table 2$)$.

\section{DISCUSSION}

The physiology and anatomy of the lung makes it difficult to obtain biological material from nodular lesions by biopsy and/or needle aspiration [20]. FNA is a well 
Table 1: Distribution of FNA specimen types according to histological diagnosis

\begin{tabular}{|c|c|c|c|c|c|}
\hline & \multirow[t]{3}{*}{ No. $(\%)$} & \multirow[t]{3}{*}{ Diagnosis } & \multirow[t]{3}{*}{ No. $(\%)$} & \multicolumn{2}{|c|}{ Type of FNA } \\
\hline & & & & \multirow{2}{*}{$\frac{\text { EBUS-TBNA }}{\text { No. } 201}$} & \multirow{2}{*}{$\frac{\text { TBNA }}{\text { No. } 72}$} \\
\hline & & & & & \\
\hline \multirow{7}{*}{ Lung tumors } & \multirow{7}{*}{$131(48)$} & Adenocarcinoma & $80(61)$ & 46 & 34 \\
\hline & & Squamous cell carcinoma & $23(18)$ & 9 & 14 \\
\hline & & Small cell carcinoma & $19(15)$ & 15 & 4 \\
\hline & & Adenosquamous carcinoma & $4(3)$ & 1 & 3 \\
\hline & & Neuroendocrine large cell carcinoma & $3(2)$ & 1 & 2 \\
\hline & & Undifferentiated carcinoma & $1(1)$ & 0 & 1 \\
\hline & & Anaplastic large cell carcinoma & $1(1)$ & 1 & 0 \\
\hline \multirow{11}{*}{$\begin{array}{l}\text { Non lung } \\
\text { tumors }\end{array}$} & \multirow{11}{*}{$22(8)$} & Mesothelioma & $1(5)$ & 1 & 0 \\
\hline & & Thyroid papillary carcinoma & $1(5)$ & 1 & 0 \\
\hline & & Ductal breast carcinoma & $2(9)$ & 2 & 0 \\
\hline & & Oropharingeal squamous carcinoma & $1(5)$ & 1 & 0 \\
\hline & & Thymoma & $1(5)$ & 1 & 0 \\
\hline & & Ovarian carcinoma & $1(5)$ & 1 & 0 \\
\hline & & Prostate adenocarcinoma & $2(9)$ & 2 & 0 \\
\hline & & Kidney adenocarcinoma & $3(14)$ & 3 & 0 \\
\hline & & Colon adenocarcinoma & $5(23)$ & 5 & 0 \\
\hline & & Lymphoma & $3(14)$ & 3 & 0 \\
\hline & & Sarcoma & $2(9)$ & 2 & 0 \\
\hline \multirow{4}{*}{ Other } & \multirow{4}{*}{$120(44)$} & Reactive lymph nodes & $90(75)$ & 90 & 0 \\
\hline & & Microgranulomatous lymphadenitis & $15(13)$ & 15 & 0 \\
\hline & & Necrosis & $1(1)$ & 1 & 0 \\
\hline & & Healthy & $14(12)$ & 0 & 14 \\
\hline
\end{tabular}

FNA = fine-needle aspiration; EBUS-TBNA = endobronchial ultrasound-transbronchial needle aspiration

tolerated diagnostic method that permits diagnostic material to be removed from a solid lesion. Although an invasive procedure, it is almost always risk-free. The majority of peripheral lung nodules and peribronchial/mediastinal lymph nodes can only be evaluated by TBNA and EBUS-TBNA, respectively [9-11]. Thus, adequate cytological material in terms of both quality and quantity is essential for diagnosis and for providing biological information that can be used to tailor therapy. Several studies have already demonstrated that cytological material is suitable for molecular analysis [2-4].

Some authors have reported diagnostic difficulties and pitfalls of ROSE for material obtained by EBUSTBNA [21-23]. In their paper, "The petals and thorns of ROSE (rapid on-site evaluation)", da Cunha Santos et al. reported an improvement in sensitivity and the need for less additional sampling (with a consequently lower risk for the patient), but also highlighted the importance of having an experienced on-site cytopathologist [21]. Moreover, several authors have also evaluated the potential correlation between ROSE and the final histological diagnosis [24-27]. However, there are still no "user-friendly" criteria to adopt during ROSE to histologically classify cytological specimens and a multidisciplinary approach involving a pulmonologist, radiologist and cytopathologist is required [26, 27].

Although we were not able to classify all lung cancer types, we demonstrated that it was possible to establish the histological type of the most frequent lung tumors during ROSE using a few easily identifiable cytomorphological features. Another weak point of our work was its retrospective nature and the low number of patients with squamous cell carcinoma or small cell lung cancer. However, the numbers reflect the normal distribution and prevalence of these tumor histotypes in a population of patients with lung cancer. 


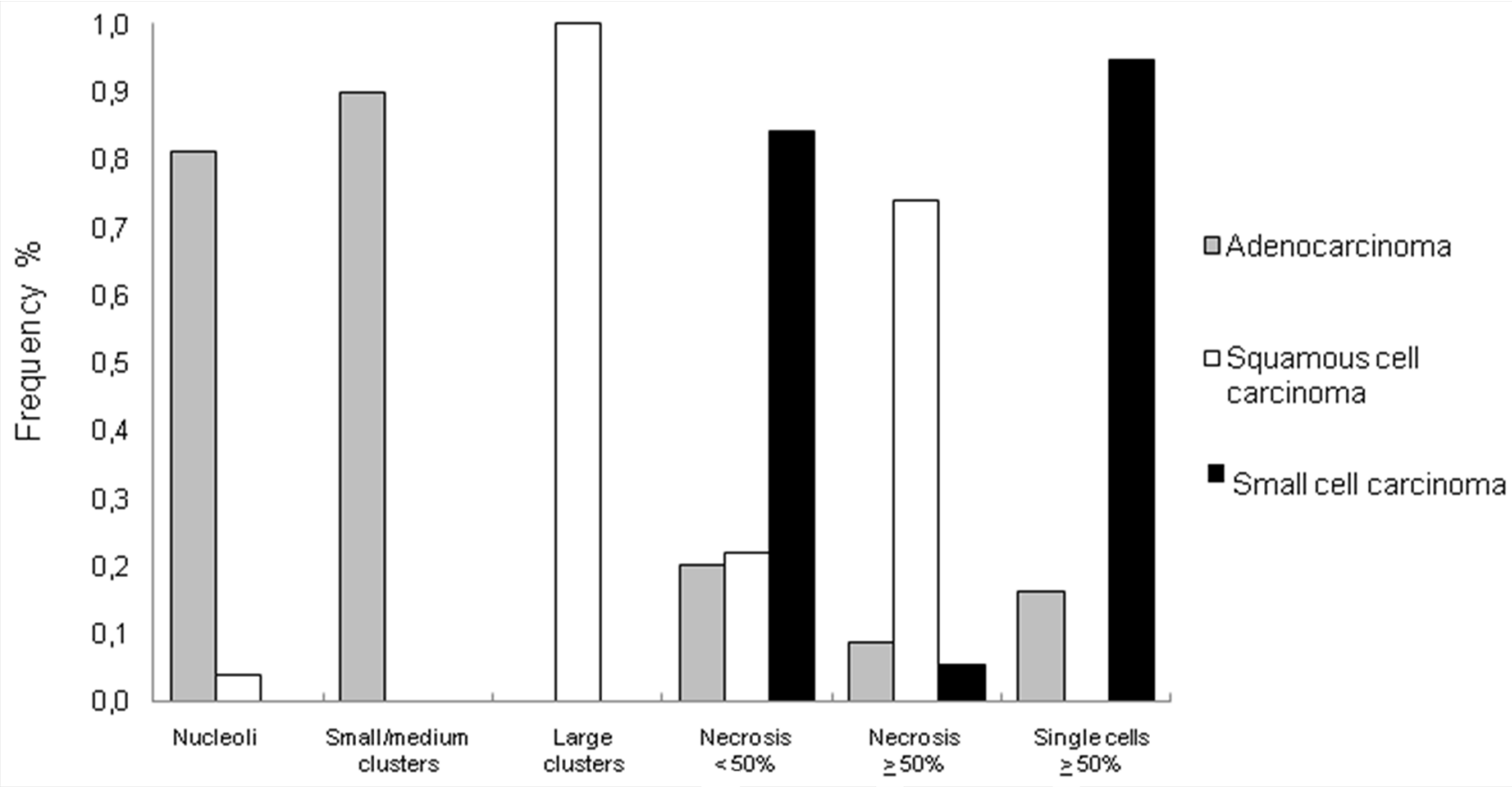

Figure 1: Frequency of cytomorphological features in different lung cancer histotypes.

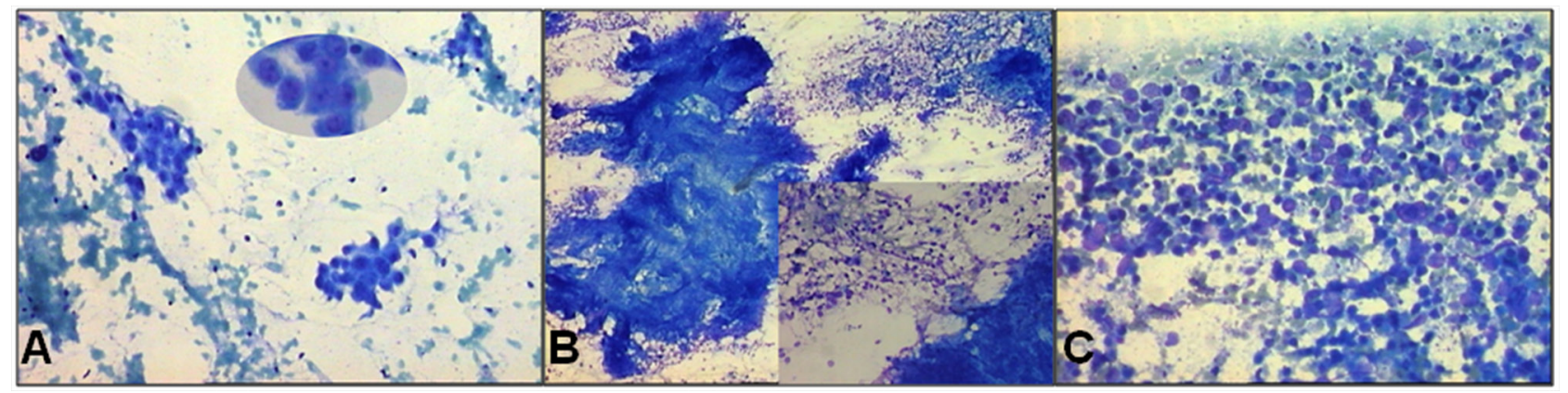

Figure 2: Cytological smears stained with fast quick MGG. A. A typical histological pattern of adenocarcinoma (10X magnification) with evident nucleoli (visible in the oval field at 20X magnification) and small/medium cell clusters. B. A typical histological pattern of squamous cell carcinoma (5X magnification) with large cell clusters and abundant necrosis (visible in the square at $10 \mathrm{X}$ magnification). C. A typical histological pattern of small cell carcinoma with necrosis and single cells (10X magnification).

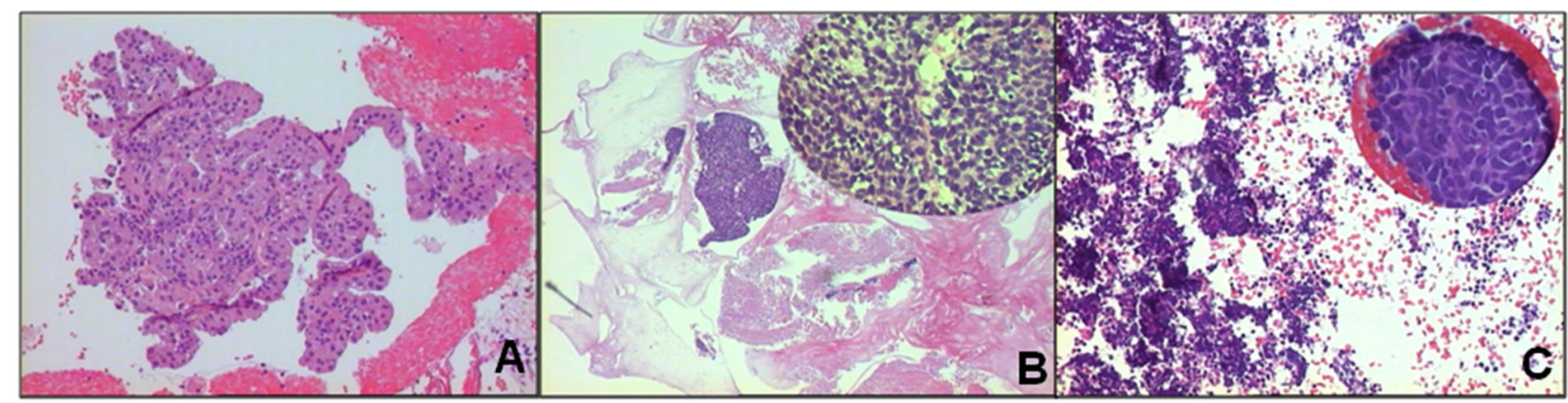

Figure 3: FFPE cytological specimens stained with hematoxylin-eosin for the final diagnosis. A. Adenocarcinoma histotype (10X magnification). B. Squamous cell carcinoma histotype (10X magnification). C. Small cell carcinoma histotype (10X magnification). 
Table 2: Diagnostic accuracy of cytomorphological features in relation to tumor histotype

\begin{tabular}{|c|c|c|c|c|c|}
\hline Histotype & Cytomorphological features & Sensitivity \% & Specificity \% & $\begin{array}{c}\text { Overall } \\
\text { Accuracy \% }\end{array}$ & $p$ \\
\hline Adenocarcinoma & $\begin{array}{l}\text { Nucleoli and small-medium } \\
\text { clusters }\end{array}$ & 73 & 98 & 90 & $<0.001$ \\
\hline $\begin{array}{l}\text { Squamous cell } \\
\text { carcinoma }\end{array}$ & High necrosis and large clusters & 74 & 99 & 97 & $<0.001$ \\
\hline $\begin{array}{l}\text { Small cell } \\
\text { carcinoma }\end{array}$ & $\begin{array}{l}\text { Moderate necrosis and single } \\
\text { cells }\end{array}$ & 79 & 96 & 94 & $<0.001$ \\
\hline
\end{tabular}

The adenocarcinoma histotype was characterized by the presence of one or more nucleoli and small/medium cell clusters, both of which showed high specificity and sensitivity. Necrosis was observed in some cases but did not exceed $50 \%$ of the cytological samples. The presence of abundant necrosis was an indication of metastasis from adenocarcinoma of the colon, breast or prostate. Large clusters were observed in almost all squamous cell carcinomas, often characterized by abundant necrosis ( $\geq 50 \%)$. In contrast, moderate necrosis $(<50 \%)$ observed together with the single cell parameter was indicative of a small cell carcinoma histotype.

An accurate diagnosis obtained by ROSE helps to define the number of samples to be taken. In cases of squamous cell or small cell carcinoma, a single sample may be sufficient, whereas multiple samples are needed for adenocarcinoma to permit biomolecular characterization. We demonstrated that a rapid lung cancer diagnosis was feasible during ROSE as it not only enabled us to establish whether the amount of aspirated material was sufficient but also identified the tumor histotype. Given that not all centers have staff trained in cytopathology, the ROSE procedure has also been taught to other professionals, e.g. pulmonologists $[27,28]$. Our findings could thus be used to train unskilled staff in histotype diagnosis via ROSE as in-depth knowledge of cell morphology is not required.

\section{MATERIALS AND METHODS}

In this retrospective study, cytological specimens were analyzed from 273 consecutive patients who underwent bronchoscopy with TBNA $(n=72)$ or EBUSTBNA $(n=201)$ at Santa Maria delle Croci Hospital in Ravenna (Italy) between January 2011 and December 2012. The study protocol was reviewed and approved by the institutional ethics committee (Ethics Committee of Area Vasta Romagna, approval no. 611). Written informed consent was obtained from all participants. For each patient, age and sex, site of fine-needle aspiration, original ROSE report (cell block status (yes/no), final diagnosis, and clinical/surgical follow-up (if available) were recorded.

The instrumental analyses were performed by the pulmonologist in the operating theater in the presence of the pathologist, nurses and the anesthesiologist. Lung lesions were evaluated by video-fiberscope (PENTAX EB1570K). TBNA biological specimens were obtained by Wang (21-22 gauge) transbronchial cytology needles under fluoroscopic guidance. Patients undergoing TBNA were mildly sedated with midazolam (Accord Healthcare Limited, Middlesex, UK) and local lidocaine (Bioindustria, L.I.M, Novi Ligure, Italy).

Mediastinal/hilar lymph node lesions and peribronchial lesions were analyzed by echo-endoscopy (EBUS PENTAX, Miyagi Factory HOYA Corporation, Japan). Lymph node stations 7, R4/L4, R2/L2 and 10 were evaluated by EBUS, while stations 5, 8 and 9 were assessed by endoscopic ultrasonography.

Twenty-two-gauge fine needles (Medi-Globe $\mathrm{GmbH}$, Germany) were used in patients under general anesthesia by laryngeal mask airway intubation. A part of the first cytological sample was smeared by the pathologist on a positively charged slide for analysis with ROSE. The remaining material was fixed in $10 \%$ formalin and embedded in paraffin to obtain a tissue block. The quantity and quality of the samples were established for each patient by ROSE.

All cytological smears were immediately fixed using 80\% alcohol and stained with Fast Quick-MayGrunwald Giemsa (MGG) (Diapath Spa, Martinengo, Italy). The entire procedure took 30/40 seconds. The slides were placed directly under the microscope (Axioskop, Carl Zeiss, Gottïngen, Germany) for analysis at $5 \mathrm{X}$ and $10 \mathrm{X}$ magnification. In the event of insufficient and/or non-diagnostic material, a part of the subsequent sample was used to obtain an adequate cytological smear for ROSE. When ROSE was feasible, at least 2 or 3 samples were taken from the same anatomic site and the material obtained was paraffin-embedded for use in ancillary assays and biomolecular investigations. The final diagnosis was made by 2 pathologists on the FFPE material of all the cytological specimens stained with hematoxylin-eosin using immunohistochemistry (TTF1, P40/P63, CK7, CK5/6, synaptophysin and chromogranin A). The diagnosis was further confirmed on the tumor tissue obtained by biopsy during broncoscopy and/or on resected tumor tissue in operable lesions.

In addition to evaluating the quality of the cytological samples, 3 easily identifiable cytomorphological features 
were analyzed on site by the pathologist and the information was recorded in a specific form for each patient:

a) Presence of one or more nucleoli.

b) Cellular organization (cells arranged in small/ medium or large clusters or the presence of individual non cluster-forming cells). Groups of cells visualized in a microscope field at 10X magnification were defined as small/medium clusters. In contrast, cells not visualized in a field at 10X magnification were classified as large clusters.

c) Amount of necrosis $(<50 \%$ or $\geq 50 \%)$ in the entire cytological sample.

These parameters were retrospectively analyzed and compared with the final diagnosis made on the FFPE sample.

\section{Statistical analyses}

The relation between cytomorphological features and tumor histotype was assessed by the chi-square test and corrected with Yates correction [29]. On the basis of the distribution of the different characteristics, sensitivity and specificity were determined by calculating the true-positive (sensitivity) and false-positive (1-specificity) rates. For all tests, a 2 -sided $P=0.05$ was regarded as significant.

\section{Abbreviations}

FNA, fine needle aspiration; ROSE, rapid on site evaluation; TBNA, transbronchial needle aspiration; EBUS, endobronchial ultrasound; FFPE, formalin-fixed paraffin-embedded; IHC, immunohistochemistry; MGG, May-Grunwald Giemsa; TTF1, thyroid transcription factor 1; CK, cytokeratin; CT, computed tomography.

\section{ACKNOWLEDGMENTS}

The authors would like to thank Cristiano Verna for editorial assistance.

\section{CONFLICTS OF INTEREST}

The authors confirm that no potential conflicts of interest exist with any companies/organizations whose products or services may be discussed in this article.

\section{FUNDING}

This research did not receive any specific grant from funding agencies in the public, commercial, or not-forprofit sectors.

\section{REFERENCES}

1. Micames CG, McCrory DC, Pavey DA, Jowell PS, Gress FG. Endoscopic ultrasound-guided fine-needle aspiration for non-small cell lung cancer staging: a systematic review and meta analysis. Chest. 2007; 131:539-548.

2. Ulivi P, Zoli W, Chiadini E, Capelli L, Candoli P, Calistri D, Silvestrini R, Puccetti M. EGFR and K-RAS mutations in cytologic samples from fine-needle aspirates in NSCLC patients. Eur Respir J. 2012; 40:267-269.

3. Wang S, Yu B, Ng CC, Mercorella B, Selinger CI, O'Toole SA, Cooper WA. The suitability of small biopsy and cytology specimens for EGFR and other mutation testing in non-small cell lung cancer. Transl Lung Cancer Res. 2015; 4:119-125.

4. Bravaccini S, Tumedei MM, Ulivi P, Zoli W, Calistri D, Candoli P, Amadori D, Puccetti M. ALK translocation detection in non-small cell lung cancer cytological samples obtained by TBNA or EBUS-TBNA. Cytopathology. 2016; 27:103-107.

5. Shure D. Fiberoptic bronchoscopy: diagnostic applications. Clin Chest Med. 1987; 8:1-13.

6. Herth F, Becker HD, Ernst A. Conventional vs endobronchial ultrasound-guided transbronchial needle aspiration: a randomized trial. Chest. 2004; 125:322-325.

7. Gasparini S, Silvestri GA. Usefulness of transbronchial needle aspiration in evaluating patients with lung cancer. Thorax. 2005; 60:890-891.

8. Vincent BD, El-Bayoumi E, Hoffman B, Doelken P, DeRosimo J, Reed C, Silvestri GA. Real-time endobronchial ultrasound-guided transbronchial lymph node aspiration. Ann Thorac Surg. 2008; 85:224-230.

9. Holty JE, Kuschner WG, Gould MK. Accuracy of transbronchial needle aspiration for mediastinal staging of non-small cell lung cancer: a meta-analysis. Thorax. 2005; 60:949-955.

10. Trisolini R, Cancellieri A, Tinelli C, Paioli D, Scudeller L, Casadei GP, Parri SF, Livi V, Bondi A, Boaron M, Patelli M. Rapid on-site evaluation of transbronchial aspirates in the diagnosis of hilar and mediastinal adenopathy: a randomized trial. Chest. 2011; 139:395-401.

11. Herth FJ, Eberhardt R, Vilmann P, Krasnik M, Ernst A. Real-time endobronchial ultrasound guided transbronchial needle aspiration for sampling mediastinal lymph nodes. Thorax. 2006; 61:795-798.

12. Gasparini S. It is time for this 'ROSE' to flower. Respiration. 2005; 72:129-131.

13. Mazza E, Maddau C, Ricciardi A, Falchini M, Matucci M, Ciarpallini T. On-site evaluation of percutaneous CT-guided fine needle aspiration of pulmonary lesions. A study of 321 cases. Radiol Med. 2005; 110:141-148.

14. Cardoso AV, Neves I, Magalhaes A, Sucena M, Barroca H, Fernandes $\mathrm{G}$. The value of rapid on-site evaluation during EBUS-TBNA. Rev Port Pneumol. 2015; 21:253-258.

15. Jeffus SK, Joiner AK, Siegel ER, Massoll NA, Meena N, Chen C, Post SR, Bartter T. Rapid on-site evaluation of EBUS-TBNA specimens of lymph nodes: Comparative 
analysis and recommendations for standardization. Cancer Cytopathol. 2015; 123:362-372.

16. Schmidt RL, Witt BL, Lopez-Calderon LE, Layfield LJ. The influence of rapid onsite evaluation on the adequacy rate of fine-needle aspiration cytology: a systematic review and meta-analysis. Am J Clin Pathol. 2013; 139:300-308.

17. Alsharif M, Andrade RS, Groth SS, Stelow EB, Pambuccian SE. Endobronchial ultrasound-guided transbronchial fineneedle aspiration: the University of Minnesota experience, with emphasis on usefulness, adequacy assessment, and diagnostic difficulties. Am J Clin Pathol. 2008; 130:434-443.

18. Kubik MJ, Bovbel A, Goli H, Saremian J, Siddiqi A, Masood S. Diagnostic value and accuracy of imprint cytology evaluation during image-guided core needle biopsies: Review of our experience at a large academic center. Diagn Cytopathol. 2015; 43:773-779.

19. Idowu MO, Powers CN. Lung cancer cytology: potential pitfalls and mimics - a review. Int J Clin Exp Pathol. 2010; 3:367-385.

20. Fassa L. Imaging and cancer: A review. Molecular Oncology 2. 2008; 115-152.

21. da Cunha Santos G, Ko HM, Saieg MA, Geddie WR. "The petals and thorns" of ROSE (rapid on-site evaluation). Cancer Cytopathol. 2013; 121:4-8.

22. Monaco SE, Schuchert MJ, and Khalbuss WE. Diagnostic difficulties and pitfalls in rapid on-site evaluation of endobronchial ultrasound guided fine needle aspiration. Cytojournal. 2010; 14:7-9.
23. Hamilton HH, Pellicier J, Bernstein M, Dimashkieh H, Yang J. Endobronchial ultrasound-guided transbronchial fine needle aspiration: advantages and potential pitfalls. Path Lab Med Int. 2015; 7:83-93.

24. Mfokazi A, Wright CA, Louw M, Von Groote-Bidlingmaier F, Schubert PT, Koegelenberg CF, Diacon AH. Direct Comparison of Liquid- Based and Smear-Based Cytology With and Without Rapid On-Site Evaluation for Fine Needle Aspirates of Thoracic Tumors. Diag Cytopathol. 2016; 44:363-368.

25. Chandra S, Chandra H, Sindhwani G. Role of rapid on-site evaluation with cyto-histopathological correlation in diagnosis of lung lesion. J Cytol. 2014; 31:189-193.

26. Burlingame OO, Kessé KO, Silverman SG, Cibas ES. On-site adequacy evaluations performed by cytotechnologists: Correlation with final interpretations of 5241 image-guided fine-needle aspiration biopsies. Cancer Cytopathol. 2012; 120:177-184.

27. Blackhall F, Thatcher N, Booton R, Kerr K. The impact on the multidisciplinary team of molecular profiling for personalized therapy in non-small cell lung cancer. Lung Cancer. 2013; 79:101-103.

28. Bonifazi M, Sediari M, Ferretti M, Poidomani G, Tramacere I, Mei F, Zuccatosta L, Gasparini S. The role of the pulmonologist in rapid on-site cytologic evaluation of transbronchial needle aspiration: a prospective study. Chest. 2014; 145:60-65.

29. Yates, F. Tests of significance for $2 \times 2$ contingency tables. J R Stat Soc Series A. 1984; 147:426-63. 